\title{
Supercontinuum-based nondisruptive scattering analyses of mouse fibroblast L929 cells before and after necrosis
}

Tobias Baselt

Alexander Kabardiadi-Virkovski

Daniel Ruf

Bryan Nelsen

Andrés Fabián Lasagni

Peter Hartmann 


\title{
Supercontinuum-based nondisruptive scattering analyses of mouse fibroblast L929 cells before and after necrosis
}

\author{
Tobias Baselt, ${ }^{a, b, c, \star}$ Alexander Kabardiadi-Virkovski, ${ }^{a, b}$ Daniel Ruf, ${ }^{a, b}$ Bryan Nelsen, ${ }^{a, b}$ Andrés Fabián Lasagni, ${ }^{c}$ \\ and Peter Hartmann ${ }^{a, b}$ \\ ${ }^{a}$ Fraunhofer-Institut für Werkstoff- und Strahltechnik IWS, Dresden, Germany \\ bUniversity of Applied Sciences Zwickau, Leupold Institute for Applied Natural Sciences/Optical Technologies, Zwickau, Germany \\ ${ }^{\mathrm{C} T e c h n i s c h e}$ Universität Dresden, Institute of Manufacturing Technology, Dresden, Germany
}

\begin{abstract}
The scattering properties of biological tissue are highly dependent on the structure size, refractive index, and wavelength of the incident light. Furthermore, these scattering characteristics are strongly influenced by movements of the scattering objects. A method is developed to determine the angular- and spectral-resolved scattering properties that enabled the characterization of biological nano- and microscaled cell structures. Nanosecond pulses from a spectrally filtered supercontinuum light source are captured and time-resolved to depress background noise and minimize disruptive effects of the biological cells. The scattering characteristics of a monolayer of mouse fibroblast L929 cells are measured at defined wavelengths in a standard cell culture plate. Because of the size and distribution of the scattering structures, a Fourier transform-based Mie scattering scheme is used to analyze the data. The system is tested to detect structural changes of mouse fibroblast L929 cells before and after poisoning with Triton X100. The final result is the development of a contamination-free method to study pathological changes in cell cultures, necrosis, or other cell-damaging effects. $\odot$ The Authors. Published by SPIE and CLP under a Creative Commons Attribution 4.0 Unported License. Distribution or reproduction of this work in whole or in part requires full attribution of the original publication, including its DOI. [DOI: 10.1117/1.JBO.23.12.121619]

Keywords: scattering analyses; supercontinuum; L929 cells; Mie scattering.
\end{abstract}

Paper 180398SSR received Jul. 2, 2018; accepted for publication Oct. 29, 2018; published online Dec. 19, 2018.

\section{Introduction}

Optical properties of living tissue are important for many diagnostic methods in the field of biomedical optics and biophotonics $^{1,2}$ and light scattering often limits the ability to analyze cellular structures. ${ }^{3,4}$ Scattering in biological tissue occurs mainly in the forward direction with the resulting angle-dependent intensity distribution being determined by the structure size, the refractive index, and the geometry of the structures. ${ }^{5}$ The random spatial distribution of the cell structures and their size distribution will also determine the scattering characteristics. All constraints must, therefore, be considered for the propagation of light in three-dimensional (3-D) biological structures, which justifies the complexity of the corresponding models. $^{6}$

The determination of structural changes in 3-D biological tissues is made possible by different imaging techniques. ${ }^{1,3,7}$ Measurements of elastic scattering of light with single cells allow a characterization of cellular structural changes. ${ }^{8,9}$ In many abnormal cell changes, the evaluation of structural changes in individual cells is not sufficient and a large number of cells must be examined to verify the results. ${ }^{10}$ Angular-dependent light scattering analyses in a suspension of cells with a concentration between $1 \times 10^{5}$ and $2 \times 10^{5}$ cells $/ \mathrm{mL}$ were realized to analyze phases of the cancer cell cycle. ${ }^{11}$ For appropriate measurements, the cells were prepared accordingly and special cuvettes were used. ${ }^{12,13}$ The use of optical radiation as a measurement tool could alter or harm the cell culture, which limits

*Address all correspondence to: Tobias Baselt, E-mail: tobias.baselt@fhzwickau.de the maximal usable intensity and measuring time. ${ }^{14}$ We have developed a nondisruptive fast method of angle-resolved scattering measurement in cell culture plates, which enables the characterization of pathological cell changes during cultivation without influencing their growth. The pseudorandom scattering characteristics were attributed to particular cellular structures and used as a method to determine the state of induced necrosis of the cells.

\section{Experimental Arrangement and Methods}

\subsection{Light-Scattering Measurements}

To investigate the spectral- and angle-resolved scattering characteristics of biological nano- and microscaled cell structures, the scattered portion of a pulsed supercontinuum (SC) light source was recorded after the sample in a time-resolved manner at defined angles. An optical setup to detect these signals was developed as shown in Fig. $1 .^{15} \mathrm{~A}$ spectrally filtered and collimated SC light source, developed and manufactured in cooperation with fiberware $\mathrm{GmbH}$, was used. ${ }^{16}$ The SC light source emits 1.1-ns pulses, a repetition rate of $8 \mathrm{kHz}$, and was filtered to a spectral bandwidth between 450 and $650 \mathrm{~nm}$.

The bandpass filters with a spectral transmission width of $10 \mathrm{~nm}$ (full width at half maximum) were integrated in a digitally controllable filter wheel to automate the measurement process. A polarizer ensured a polarization perpendicular to the detection plane. To capture the time-resolved signal independently of the signal strength, an optical trigger was used, which enables the averaging of 200 individual signals at every angular position. Each angular position was detected 


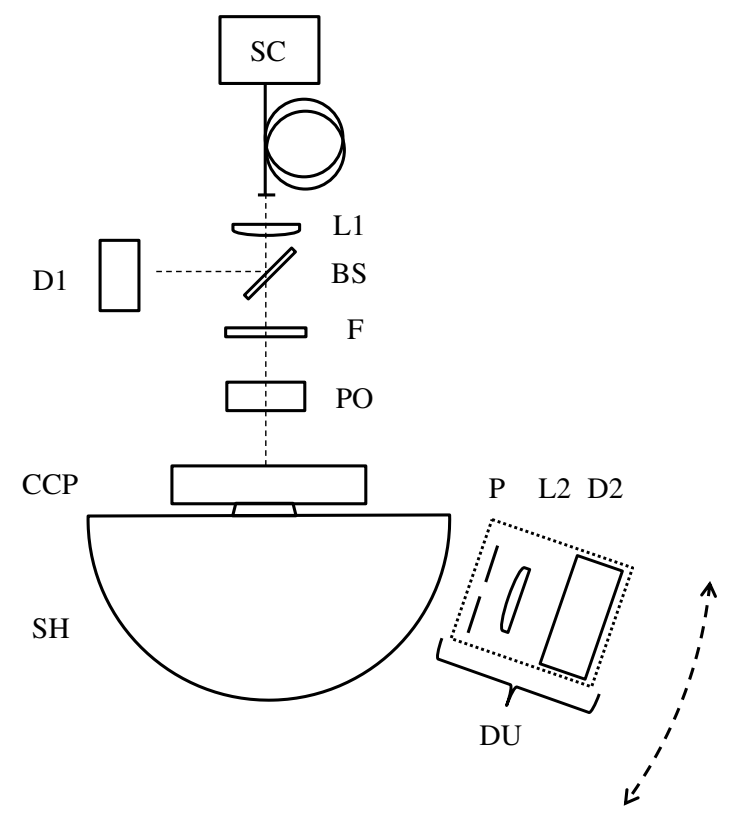

Fig. 1 Setup used to measure angular-dependent light scattering. SC, supercontinuum light source; L1, collimation lens; BS, nonpolarizing beam splitter; $\mathrm{D} 1$, reference detector; $\mathrm{F}$, bandpass filter; $\mathrm{PO}$, polarizer; $\mathrm{CCP}$, cell culture plate; $\mathrm{SH}$, specimen holder; $\mathrm{P}$, pinhole; L2, collecting lens; D2, avalanche detector; and DU, detector unit.

automatically in $<1 \mathrm{~s}$ and the complete measuring range with a $1 \mathrm{deg}$ angular resolution for each wavelength was detected in $<2.5 \mathrm{~min}$. With the automated filter change, the entire multispectral signal can be detected in $<12 \mathrm{~min}$. A standard cell culture plate was placed on the sample holder and 10 of the 24 wells were examined. Through the application of index-matching immersion gel between the cell culture plate and the specimen holder, the adapted sample holder can allow a measurement of the scattered components of the incident light in the angular range of \pm 80 deg without angle-dependent Fresnel reflection losses. The angular resolution is $\pm 1.4 \mathrm{deg}$, which is due to the beam diameter on the sample and aberrations in the collimating lens. By varying the position of detector unit, it is possible to capture the angular-dependent scattered light at a defined wavelength. The movement of the detector unit was performed automatically by a motorized rotation stage. To capture the time-resolved scattered portion of the pulsed SC-light source, an avalanche detector with a bandwidth of $50 \mathrm{MHz}$ was used.

The amplitude of the SC pulse is of importance for the measurement method as it is temporally and spectrally resolved over the angular measurement. To detect the temporal amplitude of the pulse, the signals were numerically fitted with a Gaussian. Due to the bandwidth of the avalanche detector, a temporal broadening of the pulse occurs. The pulse-to-pulse stability of the SC light source was recorded at a wavelength of $500 \mathrm{~nm}$ and is normally distributed. This reference signal was also fitted with a Gaussian-shaped curve.

\subsection{Cell Culture}

The L929 mice fibroblast cells were cultivated in a standard 24 well cell culture plate using a Dulbecco's modified eagle medium/nutrient mixture Ham's F-12, which contained $10 \%$ fetal calf serum (FCS) and 1\% L-glutamine. The cells were seeded in culture wells $24 \mathrm{~h}$ before the measurement. The culture medium was incubated under a temperature of $37^{\circ} \mathrm{C}$. The humidity was saturated and the $\mathrm{CO}_{2}$ concentration was $5 \%$ in the incubator. The cell culture plates were removed from the incubator immediately prior to measurement, and the measurement time was limited to $30 \mathrm{~min}$. In the case where induced necrosis was investigated, Triton X100 was added. ${ }^{17}$

\section{Results and Discussion}

To qualify the measuring setup with angular resolution (Fig. 1), we first used polystyrene beads in a water solution as a standardized sample to examine the scattering properties of the system. Under these conditions, Mie theory can be applied and scattering distributions are calculated with the software MiePlot v4.6.12 [see Fig. 2(a)]. ${ }^{18}$ The size uniformity of the polystyrene beads with a diameter of $1.6 \mu \mathrm{m}$ was assumed to be $\pm 3 \%$ with wavelength-dependent refractive index defined for the standardized polystyrene beads in a liquid solution. ${ }^{19,20}$ The polystyrene bead solution was loaded into a standard 24-well cell culture plate to measure the angle-resolved scattering characteristics. To analyze the wavelength-dependent scattering characteristics, the position of the second minima of the scattered fringes at different wavelengths was recorded and compared with the data calculated by Mie theory, Fig. 2(b). In comparison with monochromatic setups for angle-resolved scattering measurement, ${ }^{9,11,12}$ the presented system enables the elimination of systematic errors based on a multispectral analysis of the scattering characteristic.
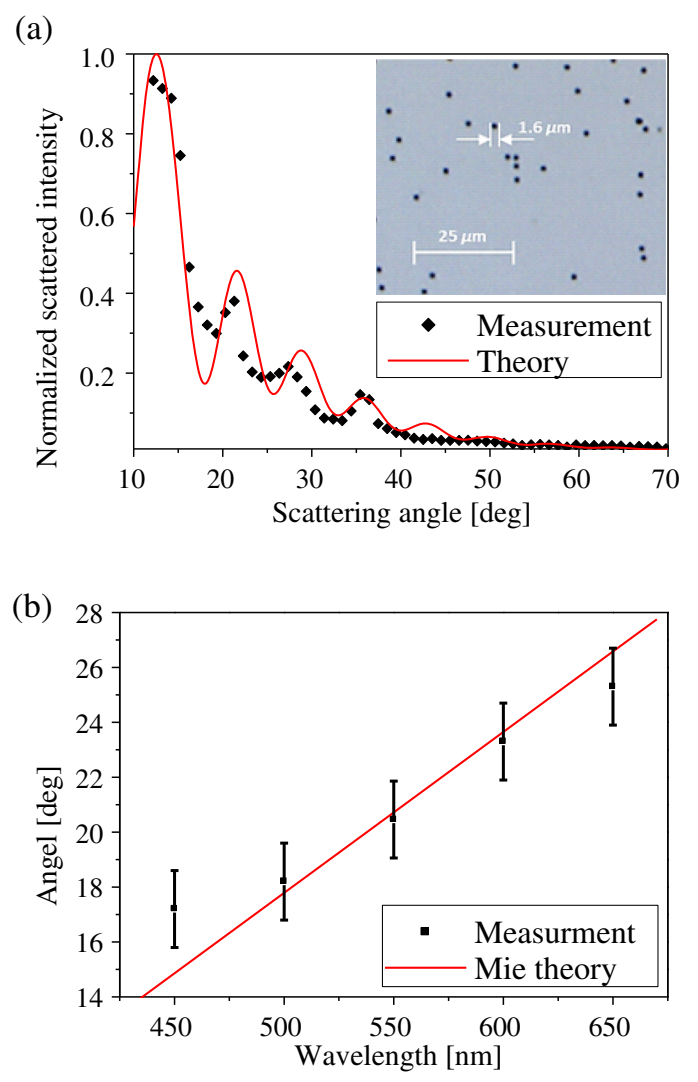

Fig. 2 (a) Angularly resolved signal of the polystyrene beads at a wavelength of $500 \mathrm{~nm}$ in a water solution with a model calculated according to Mie theory. The inset shows a microscope image of the polystyrene beads with a diameter of $1.6 \mu \mathrm{m}$. (b) Wavelength-dependent angular shift of the second minimum of the scattering fringes. 


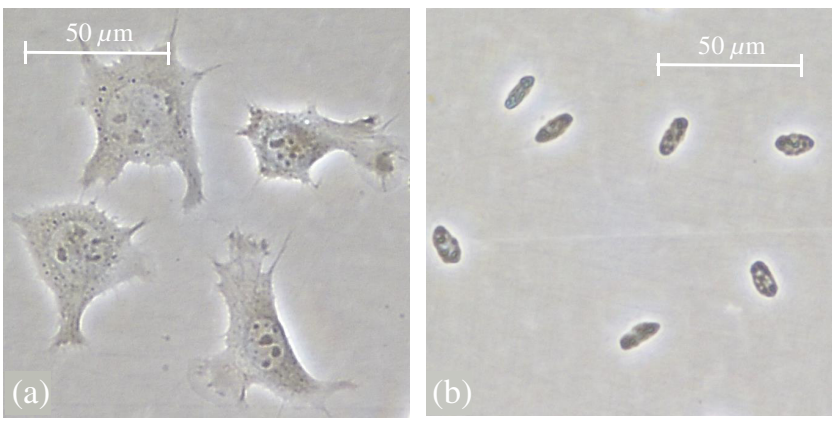

Fig. 3 Mouse fibroblast L929 cells before (a) and after necrosis (b) by adding Triton $\mathrm{X} 100 .^{14}$

To demonstrate the possibility to analyze the change of cell components after necrosis based on wavelength-dependent scattering analyses, L929 mice fibroblast cells were killed by adding Triton X100. Through the addition of Triton X100, the cell membrane is ruptured and necrosis occurs. The cells are examined by light microscopes before and after necrosis, as shown in Figs. 3(a) and 3(b), respectively. The size of the components of the cell, as well as the structures remaining after necrosis, was measured and these parameters were used as inputs for the calculations.

The cells consist of components that are fixed on the bottom of the cell culture plate as well as other movable components within the cell and nucleus. The entire population of cells $(33.8 \mathrm{~mm} \times 22.3 \mathrm{~mm} \pm 30 \%)$ is fixed on the bottom of the

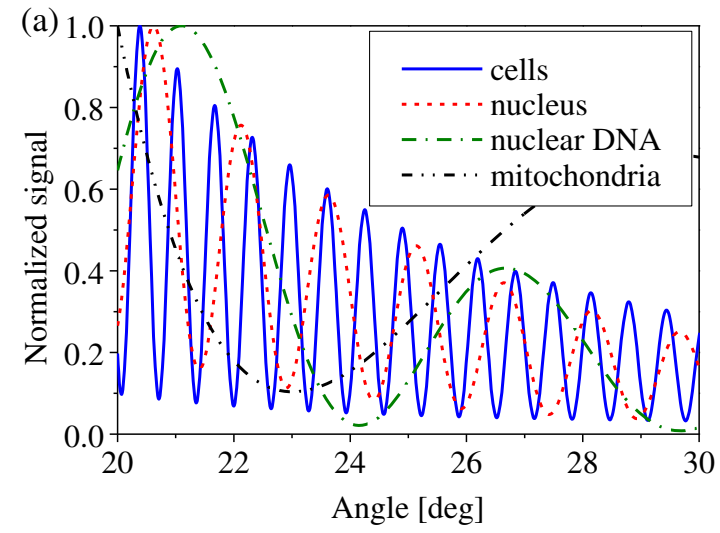

(b)

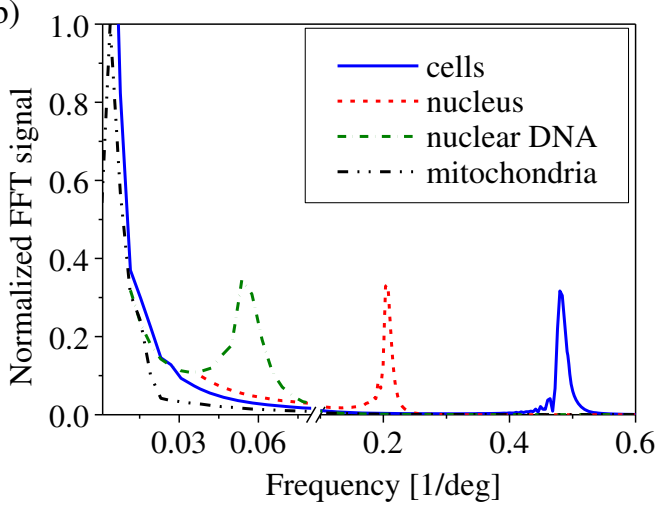

Fig. 4 (a) Calculated angularly resolved signal of the mouse fibroblast L929 cells before necrosis according to Mie theory at a wavelength $500 \mathrm{~nm}$ and (b) FFT for the calculated signals of the individual cell components. cell culture plate, and, in the measurement area with a 5-mm diameter, $3558 \pm 178$ cells are counted. To calculate the scattering properties, the aforementioned diffraction simulations were applied. Mitochondria $(n=1.42, \lambda=500 \mathrm{~nm})$ in the cell liquor $(n=1.35, \lambda=500 \mathrm{~nm})$ with a measured size of $1.28 \mu \mathrm{m} \pm 3 \%$ are randomly and uniformly distributed due to their abundance and movable arrangement. ${ }^{21}$ The nuclear components in the core of the cell have a size of $3.8 \mu \mathrm{m}$ with $20 \%$ variation. For all cell components, Mie theory was applied and the calculated angleresolved scattering characteristics at $500 \mathrm{~nm}$ for the individual cell components are shown in Fig. 4(a). Many different features of the cell, such as statistical variation in size and shape [clearly evident in Fig. 3(a)] and absorption, as well as correlations between the individual component's scattering distributions, ${ }^{22,23}$ can cause variations in the final measurement. Therefore, the relative concentration of each cell component is more relevant for determining cell viability. A spectral analysis using the fast Fourier transform (FFT) of the angle-resolved curves, ${ }^{24}$ Fig. 4(b), decouples any phase correlations between the individual cell components. The cell component's relative amplitudes, as well as their frequency shifts before and after necrosis, are a good indicator of viability.

The scattering signals of 10 different wells of a cell culture plate are characterized before and after necrosis. The background of the cell culture plate, culture medium, immersions liquid, and other optical components is captured and subtracted from the signal. The comparison of the angle-resolved scattering pattern of the cells before and after necrosis is shown in Fig. 5(a), which shows a strong shift in the structure of the
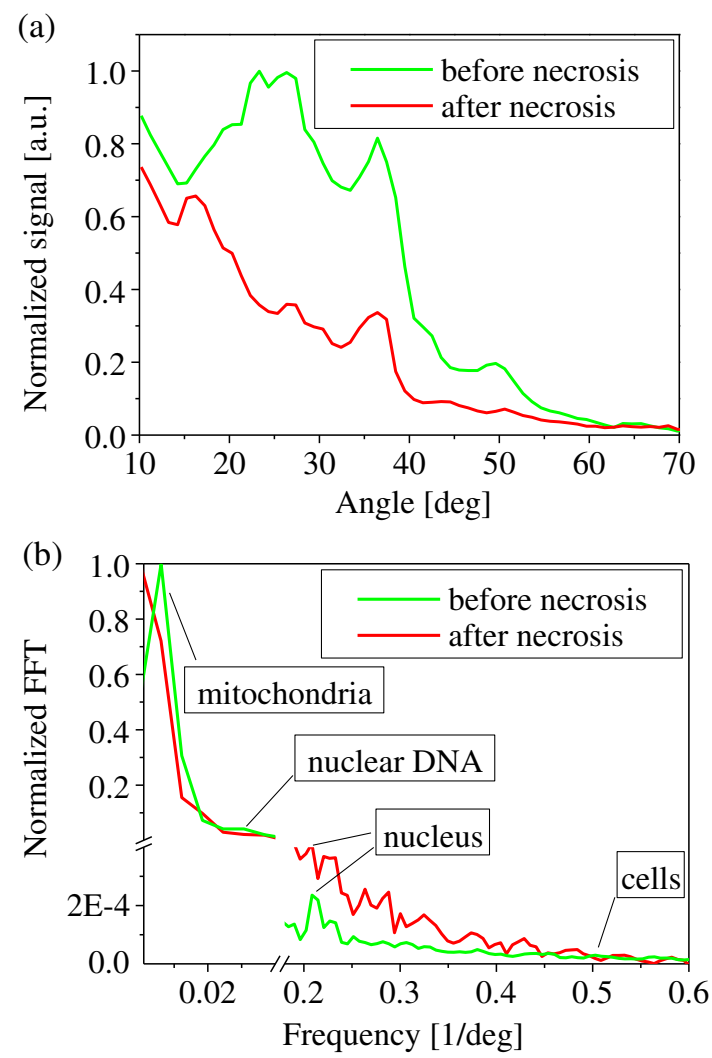

Fig. 5 (a) Angularly resolved signal of the mouse fibroblast L929 cells before and after necrosis and (b) frequency components of the angular-resolved measurement of the scattered light before and after necrosis. 


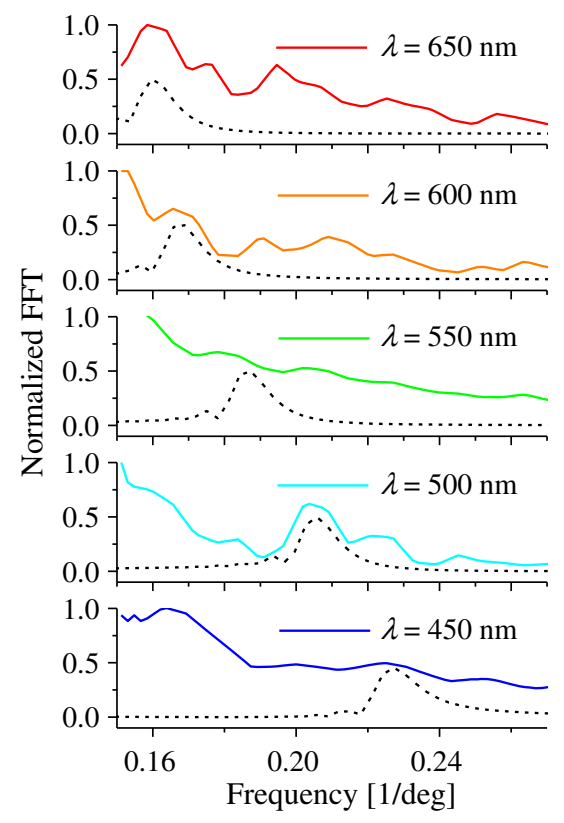

Fig. 6 Wavelength-dependent shift of the nucleus frequency peak before necrosis. The black dashed line in each plot is the theoretical curve calculated by Mie theory.

scattering intensity. The Fourier amplitude of the data in Fig. 5(a) is shown in Fig. 5(b). Due to the large size, statistical variation, and weak index contrast of the cell membrane structures, a marker is drawn where the cell membrane scattering frequency should show up due to Mie scattering but is strongly disbursed and not visible.

By destroying the cell envelope, the mitochondria are removed and that portion of scattered light disappears from the measured signal. Based on their approximate location in the frequency domain [Fig. 4(b)] as calculated by Mie theory, as well as their behavior during necrosis, peaks were labeled with their known cell components. The signal of the cells after necrosis is numerically approximated by taking scattering calculations of the shriveled nuclei into account [Fig. 3(b)].

Further verification that the assigned labels for the cell components were correct was obtained through the multispectral analysis of the selectable-wavelength SC light source. As Mie theory is known to not provide accurate results in many cases involving complicated structures such as cells, ${ }^{25}$ this second verification step was necessary to ensure the feature in the frequency domain truly behaved as expected. While the peaks of the cell components in the frequency domain shift as expected with wavelength as seen in Fig. 6, superficial frequency peaks due to noise, correlation, or redistribution of intensity among the peaks do not.

\section{Conclusion}

Nondisruptive label-free detection of cell viability in a large culture of L929 fibroblast cells was demonstrated using a multispectral angular scattering measurement system. The cells themselves present technical obstacles in scattering measurements to overcome, based on the inherent randomness of cell size and structure, and the quasicorrelated distribution, as well as optical absorption, of components within the cell. Due to these variations, Mie theory poorly predicted the angular scattering intensity patterns from these structures; however, the spectral frequencies based on the Fourier transform of the angular scattering intensity displayed critical information provided by Mie theory on the relative population of cell components before and after necrosis. Peaks were visible in the Fourier domain that correlated directly to the critical components of the cell, which change under necrosis. This method of analysis, coupled with the multispectral SC, allowed for the labeling of cell components before and after necrosis, and also provided an indicator of their relative population density. These measurements will be further implemented in a bioreactor environment as a technique to quickly and nondisruptively observe cell culture reactions to varying stimuli.

\section{Disclosures}

The authors have no relevant financial interests in this article and no potential conflicts of interest to disclose.

\section{Acknowledgments}

The authors want to thank the State Ministry for Higher Education, Research and the Arts, for financial support under Grant No. 4-7544.10/7/3 as well as the Research Institute of Leather and Plastic Sheeting-FILK for the possibility of measuring cell cultures and members of the optical technologies working group at West Saxon University of Applied Sciences, Zwickau.

\section{References}

1. S. Jiao and L. V. Wang, "Two-dimensional depth-resolved Mueller matrix of biological tissue measured with double-beam polarizationsensitive optical coherence tomography," Opt. Lett. 27(2), 101-103 (2002).

2. M. Ernstberger et al., "Spectrophotometric measurements of human tissues for the detection of subjacent blood vessels in an endonasal endoscopic surgical approach," J. Biophotonics 6(4), 310-313 (2013).

3. J. A. Izatt et al., "In vivo bidirectional color Doppler flow imaging of picoliter blood volumes using optical coherence tomography," Opt. Lett. 22(18), 1439-1441 (1997).

4. M. Ernstberger et al., "Combined spectrophotometry and tensile measurements of human connective tissues: potentials and limitations," J. Biomed. Opt. 18(6), 060506 (2013).

5. A. D. Kim and J. B. Keller, "Light propagation in biological tissue," J. Opt. Soc. Am. A 20(1), 92-98 (2003).

6. S. Michel, B. C. Patterson, and D. R. Wyman, "The propagation of optical radiation in tissue $\mathrm{I}$. Models of radiation transport and their application," Lasers Med. Sci. 6, 155-168 (1990).

7. R. A. R. Rao, M. R. Methaand, and K. C. Toussaint, "Fourier transformsecond-harmonic generation imaging of biological tissues," Opt. Express 17(17), 14534-14542 (2009).

8. V. Richter and H. Schneckenburger, "Light scattering microscopy for tumor cell recognition," ALT Proc. 1, 1.59 (2012).

9. J. R. Mourant et al., "Angular dependent light scattering from multicellular spheroids," J. Biomed. Opt. 7(1), 93-99 (2002).

10. A. L. Litvinenko et al., "Fluorescence-free flow cytometry for measurement of shape index distribution of resting, partially activated, and fully activated platelets," Cytometry Part A 89A, 1010-1016 (2016).

11. X. Lin et al., "Angular-dependent light scattering from cancer cells in different phases of the cell cycle," Appl. Opt. 56(29), 8154-8158 (2017).

12. B. V. Bronk, W. P. Van De Merwe, and M. Stanley, "In vivo measure of average bacterial cell size from a polarized light scattering function," Cytometry 13, 155-162 (1992).

13. J. Dai et al., "Measurement of the light scattering of single micrometersized particles captured with a microfluidic trap," Opt. Express 23(23), 30204-30215 (2015).

14. F. Rudek et al., "Non-disruptive measurement system of cell viability in bioreactors," Proc. SPIE 9711, 97110 Y (2016). 
15. T. Baselt et al., "Detection of structural changes based on Mie-scattering analyses of mouse fibroblast L929 cells before and after necrosis," Proc. SPIE 10685, 106854D (2018).

16. T. Baselt et al., "Application of a microchip laser-pumped photonic crystal fiber supercontinuum source for high-sensitive cavity ring down optical loss measurements," Proc. SPIE 7195, $71951 \mathrm{~T}$ (2009).

17. T. Hennet, C. Richter, and E. Peterhans, "Tumour necrosis factor- $\alpha$ induces superoxide anion generation in mitochondria of L929 cells," Biochem. J. 289(2), 587-592 (1993).

18. C. F. Bohren and D. R. Huffman, Absorption and Scattering of Light by Small Particles, Wiley-VCH, Weinheim (2004).

19. N. Sultanova, S. Kasarova, and I. Nikolov, "Dispersion properties of optical polymers," Acta Phys. Pol. A 116, 585-587 (2009).

20. G. M. Hale and M. R. Querry, "Optical constants of water in the 200-nm to 200-mm wavelength region," Appl. Opt. 12(3), 555-563 (1973).

21. S. Johnsen and E. A. Widder, "The physical basis of transparency in biological tissue: ultrastructure and the minimization of light scattering," J. Theor. Biol. 199(2), 181-198 (1999).

22. D. W. Mackowski and M. I. Mishchenko, "A multiple sphere T-matrix Fortan code for use on parallel computer clusters," J. Quant. Spectrosc. Radiat. Transfer 112(13), 2182-2192 (2011).

23. A. E. Moskalensky et al., "Additivity of light-scattering patterns of aggregated biological particles," J. Biomed. Opt. 19(8), 085004 (2014).

24. X. Su et al., "Light scattering characterization of mitochondrial aggregation in single cells," Opt. Express 17(16), 13381-13388 (2009).

25. M. A. Yurkin, V. P. Maltsev, and A. G. Hoekstra, "Convergence of the discrete dipole approximation. I. Theoretical analysis," J. Opt. Soc. Am. A 23(10) 2578-2591 (2006).

Tobias Baselt is a doctoral student working a joint position with Fraunhofer IWS, Zwickau and the Optical Technologies group at the West Saxon University of Applied Science Zwickau. Currently, he is working on his PhD from the Technical University of Dresden in the field of application of SC light sources in the field of optical metrology. During his $\mathrm{PhD}$ thesis, he is constantly working on improving SC generation and fiber characterization techniques.

Alexander Kabardiadi-Virkovski is working on his $\mathrm{PhD}$ in the field of wavefront analysis, scatter characteristics, and optoelectronics in metrology at the West Saxon University of Applied Sciences Zwickau.

Daniel Ruf is a master's student working in the Optical Technologies Group at the West Saxon University of Applied Science Zwickau.

Bryan Nelsen currently holds a joint postdoctoral research position at Fraunhofer IWS, Zwickau, and the West Saxon University of Applied Sciences Zwickau. After receiving his bachelor's degree in physics from Georgia Southern University in 2005, he went on to receive both his master's and $\mathrm{PhD}$ degrees in condensed matter physics from the University of Pittsburgh in 2008 and 2012, respectively. His current research interests lie in the area of specialty fibers and sensors, as well as holographic wavefront sensing.

Andrés Fabián Lasagni received his MS degree in chemical engineering from Comahue National University, Argentina, in 2002. From 2003 to 2005, he carried out his PhD at Universität des Saarlandes, Germany, and in 2007 to 2008 , he conducted a postdoctoral stay at Georgia Institute of Technology and the University of Michigan. Since 2008, he has been a group leader at the Fraunhofer IWS and as of 2012, he has been a professor at Technische Universität Dresden, Germany.

Peter Hartmann received his PhD from Friedrich Schiller University, Jena, in 1992. Afterward, he joined the Institute of Optics and Quantum Electronics Jena. Currently, he is a professor of optical technologies at the University of Applied Sciences Zwickau and head of the Fraunhofer Application Center of Optical Metrology and Surface Technologies. 